\title{
A new surgical technique of uterine auto-transplantation in cynomolgus monkey: preliminary report about two cases
}

\author{
Iori Kisu • Makoto Mihara $\cdot$ Kouji Banno $\cdot$ Hisako Hara • \\ Takumi Yamamoto $\cdot$ Jun Araki $\cdot$ Takuya Iida $\cdot$ Yohei Hayashi · \\ Hisashi Moriguchi $\cdot$ Daisuke Aoki
}

Received: 1 February 2011/ Accepted: 24 March 2011/Published online: 8 April 2011

(C) The Author(s) 2011. This article is published with open access at Springerlink.com

\begin{abstract}
Objective Uterine transplantation experiments have been performed in various animal species for future clinical applications of uterine transplantation for permanent uterine factor infertility in humans. The aim of this study was to confirm the feasibility of uterine auto-transplantation in cynomolgus monkeys by developing new surgical techniques.

Methods Two female cynomolgus monkeys underwent surgery under general anesthesia. The uterus with vascular grafts and the vaginal wall was removed, and back-table preparation was performed using heparinized saline. The uterus with vascular grafts and the vaginal wall was anastomosed with the vaginal stump and blood vessels in the pelvis, respectively. The auto-transplant uterine function was evaluated by confirming engraftment of the uterus by laparotomy, endometrial proliferation by transabdominal ultrasonography and periodical menstruation.

Results The first animal died due to acute renal failure 2 days after the operation. Second-look laparotomy in the second animal at 40 days after the operation indicated there was no congestion in the uterus, and the uterus showed the typical red color of a normal uterus. Thereafter, endometrial proliferation was observed by transabdominal
\end{abstract}

\footnotetext{
I. Kisu $\cdot$ K. Banno $(\bowtie) \cdot$ D. Aoki

Department of Obstetrics and Gynecology,

School of Medicine, Keio University, 35 Shinanomachi,

Shinjuku-ku, Tokyo 160-8582, Japan

e-mail:kbanno@sc.itc.keio.ac.jp

M. Mihara - H. Hara - T. Yamamoto - J. Araki - T. Iida ·

Y. Hayashi · H. Moriguchi

Department of Plastic and Reconstructive Surgery,

Graduates School of Medicine, University of Tokyo,

Tokyo, Japan
}

ultrasonography and periodical menstruation was confirmed, indicating re-established uterine function.

Conclusion This is the preliminary report of uterine autotransplantation in cynomolgus monkeys. This study demonstrates the feasibility of uterine auto-transplantation by using new surgical technique in cynomolgus monkeys. Accumulation of basic experimental data in non-human primates is required prior to performing the procedure in humans.

Keywords Uterine transplantation .

Auto-transplantation · Cynomolgus monkey ·

Uterine infertility $\cdot$ Supermicrosurgery $\cdot$ MRKH syndrome

\section{Introduction}

The recent advances in assisted reproductive technology such as hormonal stimulation, IVF, and ICSI have made it possible to circumvent many causes of male and female infertility [1]. However, since surrogacy is not approved in many countries due to legal, ethical and religious issues [2], pregnancy and delivery are not possible for women who undergo hysterectomy at a young age for malignant uterine tumors [3-5] and benign diseases such as myoma or adenomyosis [6], patients with massive blood loss after delivery and not in those with congenital uterine infertility, such as Mayer-Rokitansky-Küster-Hauser (MRKH) syndrome [7, 8], in whom the uterus and vagina are absent. This inability to bear children may greatly reduce the quality-of-life of young women. Reconstruction of fecundity by uterine transplantation was attempted in Saudi Arabia in 2002 [9], but necrosis of the graft occurred 99 days after surgery and the attempt failed. No uterine transplantation in humans has been reported thereafter. 
There have been marked advances in organ transplantation techniques, microvascular anastomosis techniques and immunosuppressants, with many cases of pregnancy and delivery after organ transplantation reported [10]. Nonvital allografts such as the hand and face have recently been reported [11], but uterine transplantation remains controversial. Transplantation of female internal genital organs was attempted by fallopian tube transplantation in the 1970s [12, 13], but this approach disappeared after the success of IVF. Thereafter, research in reproductive organ transplantation like ovaries has continued.

Uterine transplantation experiments have been performed in various animal species, such as the mouse [14], the rat $[15,16]$, the pig [17, 18], the sheep [19] and the baboon [20], but these have not led to successful clinical applications as a treatment for permanent uterine factor infertility in humans. The ethical guidelines established by the International Federation of Gynecology and Obstetrics (FIGO) describes that uterine transplantation, which may reach human clinical experimentation stage, should only occur after significant and adequate research in appropriate large animal models, including primates [21]. Recently, reports on uterine auto-transplantation in the baboon and allogeneic uterine transplantation in the rhesus macaque were presented $[9,20,22]$. It is important to achieve success in primates before a second human uterine transplantation is attempted, because their uterine anatomy and reproductive physiology closely resemble that of the human [23]. Moreover, cynomolgus monkeys breed and deliver offspring throughout the year, which is consistent with human reproduction.

This study prepared a uterine auto-transplantation model in cynomolgus monkeys, with the goal of establishing a fecundity reconstruction technique. This report presents the results of those experiments, focusing on the surgical procedure of auto-transplantation in cynomolgus monkeys.

\section{Materials and methods}

\section{Animals}

Two female cynomolgus monkeys with regular menstrual cycles with length generally $29.4 \pm 4.3$ days were used in this experiment. The cynomolgus monkeys belong to the Macaca (Cercopithecidae) family. This species was originally distributed in south-east Asia, including the Philippines, Sumatra, and Java, and was artificially introduced and settled in Hong Kong, Irian Jaya, Palau, Mauritius, and Samoa. The body size varies among subspecies, and the body and tail lengths are $38-55 \mathrm{~cm}$ and $40-65 \mathrm{~cm}$, respectively, in adults. The body weight is $5-9 \mathrm{~kg}$ in males and 3-6 kg in females, about one-tenth of those of humans.
The cynomolgus monkey of Case 1 was 6 years of age and the body weight was $3.56 \mathrm{~kg}$. That of Case 2 was 5 years of age and $4.21 \mathrm{~kg}$. The animal experiments in this study were performed after approval by the Ethics Committee of the experimental facility.

\section{Anesthesia}

The animals were sedated with atropine sulfate $(0.02 \mathrm{~mL} / \mathrm{kg}$ intramuscular injection) and ketamine hydrochloride $(0.1-0.2 \mathrm{~mL} / \mathrm{kg}$ intramuscular injection in a $50 \mathrm{mg} / \mathrm{mL}$ aqueous solution), the abdominal regions were shaved and the animals were positioned in the supine position. The abdominal region was disinfected with $70 \%$ ethanol and iodine tincture and covered with sterilized drapes. A tracheal tube was inserted and the anesthetic was maintained with isoflurane inhalation $(0.5-1.0 \%)$.

\section{Excision of the uterus}

All surgical procedures were performed using sterile techniques and the surgeons used surgical loupes. A midline incision from the pubic bone up to the level of umbilicus was performed. The intestines were packed into the upper abdomen.

The anatomical characteristics of the internal genital organs of the cynomolgus monkeys are similar to those of humans (Fig. 1a), and the vascular and ureteral distribution is very similar to that of humans. The ureter crosses the uterine artery on the dorsal side and reaches the urinary bladder (Fig. 1b). The round ligament was processed, and the broad ligaments of the uterus and retroperitoneum were exposed. The blood vessels and ureter were dissected, the vesico-uterine ligament was cut and tied, vascular pedicles were prepared, the paracolpium was processed, the vaginal canal was incised, and the uterus was excised. The ovaries and ovarian ducts were not excised, and only the uterus was excised. Orthotopic uterus retrieval requires the excision of the uterus including the connected blood vessel pedicles and vagina wall together, not in the same way as in a simple total hysterectomy.

\section{Selections of vascular grafts}

The vascular anatomy of the internal genital organs of the cynomolgus monkeys resembles that of the human female (Fig. 2). The vessels associated with blood flow in the uterus are the uterine arteries, superficial uterine veins, deep uterine veins, ovarian arteries and veins (Fig. 3a). The vessels, except for the ovarian arteries and veins, were selected as vascular grafts because the utero-ovarian ligaments were cut and tied bilaterally to preserve the ovaries and tubes. The deep uterine vein of the cynomolgus 
Fig. 1 Anatomical characteristics of the inner genital organs of cynomolgus monkey. The uterus, ovaries, fallopian tubes, round ligament, and urinary bladder are shown (a). The anatomical characteristics of the inner genital organs are almost the same as those in humans. The ureter crosses the uterine artery and vein on the dorsal side, as in humans (b)


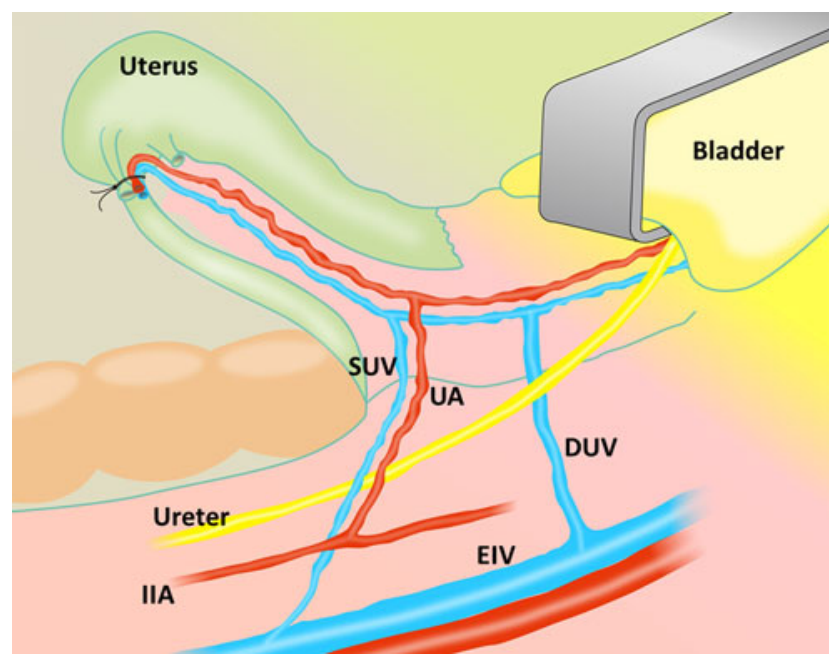

Fig. 2 Relationship between the uterus and uterine blood vessels. The main uterine artery branches from the internal iliac artery (IIA) and divides into the ascending and descending branches. The ascending branch feeds the uterine body and joins the ovarian artery. The descending branch extends to the paracolpium. The superficial uterine vein $(S U V)$ is located along the uterine artery $(U A)$. The deep uterine vein $(D U V)$ connects to the external iliac vein $(E I V)$ in the cynomolgus monkey. The ureter is located along the uterine artery on the dorsal side and reaches the urinary bladder

monkeys differs from that of the human, and is found in a deeper region of pelvis than the uterine artery and flows to the external iliac vein. The deep uterine vein could not be identified in Case 1, therefore the internal iliac arteries, uterine arteries and the superficial uterine veins were selected as vascular grafts. The deep uterine veins that run in the deep region of pelvis were identified in Case 2, and the uterine arteries and deep uterine veins were selected as vascular grafts.

\section{Excision of the vaginal wall}

The vaginal wall is minimally included in the excised uterine body in a simple total hysterectomy. To include the abundant vaginal wall in the procedure, the excision requires performance of a semi-radical hysterectomy, or a radical hysterectomy. Inclusion of the abundant vaginal wall also requires ureteral dissection and processing of the vesico-uterine ligament and cardinal ligament, thereby increasing surgical stress. The vaginal tract was cut at a shallower part in Case 1, in contrast to the deep uterine vein, and the uterus together with the vaginal wall was excised. It was cut at a deeper part in contrast to the deep uterine vein in Case 2 because the deep uterine veins were selected as vascular pedicles, which required processing of the surrounding tissue in the deep region of pelvis. Consequently, a longer vaginal wall $(14 \mathrm{~mm})$ was excised in Case 2 than that $(6 \mathrm{~mm})$ in Case 1 (Fig. $3 b)$.

\section{Back-table preparation}

The uterus was transferred to a back-table while the arteries and veins were clipped. The weight of the uterus was about $10 \mathrm{~g}$ in each case. The fluorinated plastic outer needle (outer diameter: $0.74 \mathrm{~mm}$, inner diameter: $0.52 \mathrm{~mm}$ ) of a $27 \mathrm{G}$ needle for venous placement was inserted into the uterine artery under a stereoscopic microscope at a magnification of $20 \times$. The outer needle was connected to a $1-\mathrm{cc}$ syringe filled with heparinized saline. The region of the uterine artery in which the outer needle was inserted was clipped using a clip for cerebral aneurysm (Sugita clip, Mizuho Co., Japan) to prevent the outer needle separating from the artery during perfusion. Fifty 1-cc syringes were prepared, and the uterus was perfused by sequentially attaching the 50 syringes to the outer needle to avoid thrombus formation. Consequently, the uterine color changed from reddish to whitish after perfusion (Fig. 4). The vaginal stump and retroperitoneum were closed to mimic a donor-side operation of future heterogeneous uterine transplantation. The uterus was kept on ice slush until it was transferred to surgical field.

\section{Uterine auto-transplantation}

The closed retroperitoneum was opened, and the vaginal stump was localized in the surgical field by inserting a 
Fig. 3 Uterus excised with the vaginal wall. Uterus was connected only via the bilateral uterine arteries and superficial uterine veins in Case 1 (a). The with the vascular pedicles and the vaginal wall $(11 \mathrm{~mm}$ anterior and $14 \mathrm{~mm}$ posterior) in Case 2 (b). $U C$ uterine cervix front side of the uterus excised
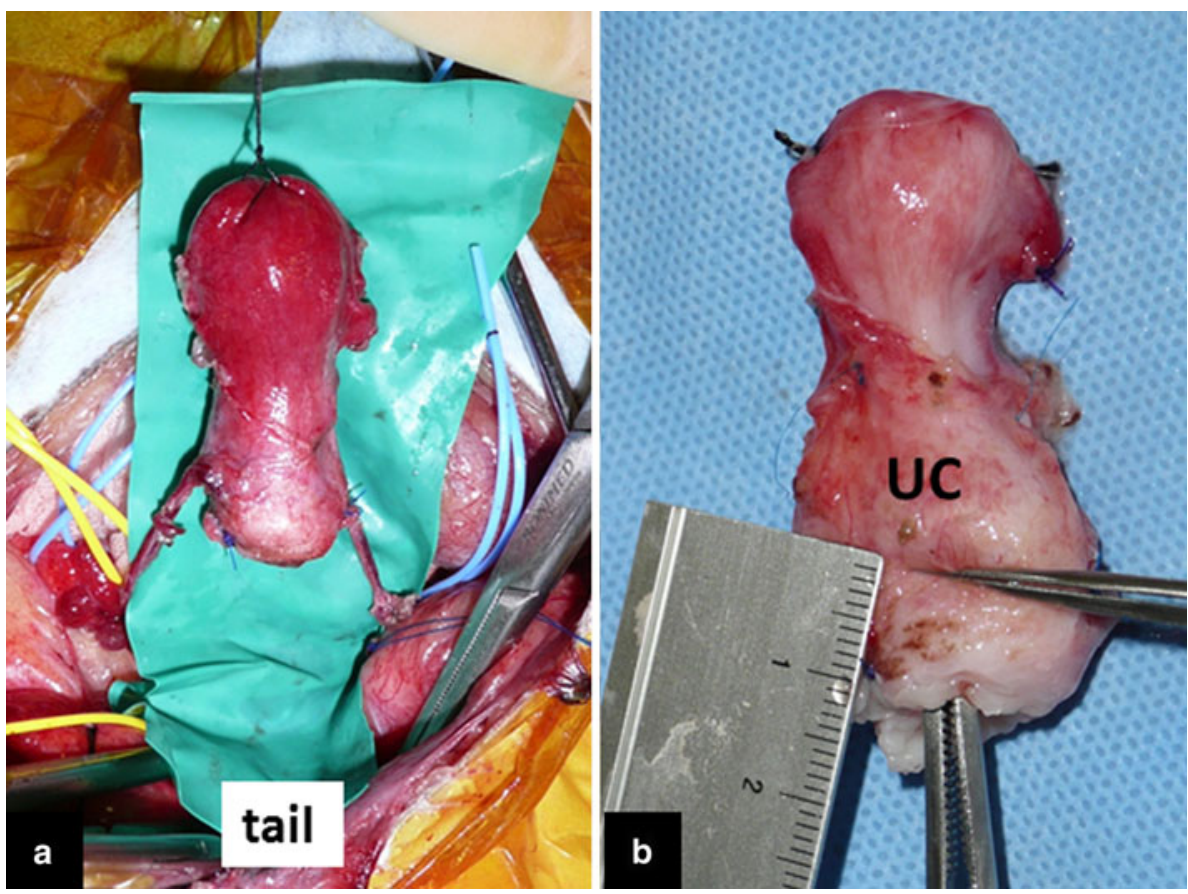

Fig. 4 The uterus before (a) and after (b) perfusion with heparinized saline via the uterine artery on the back-table. Blood components were washed out and the whole uterus became whitish in color

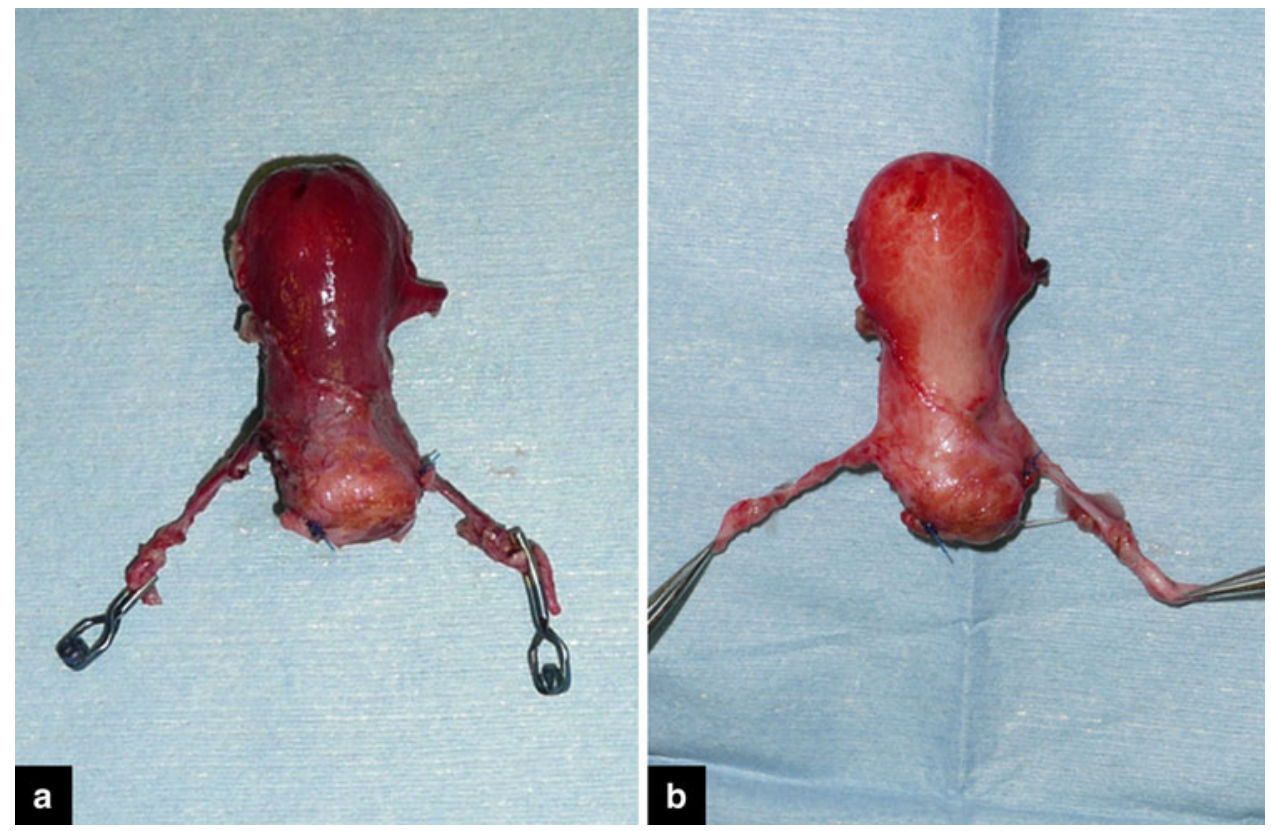

suction tube via the vagina and opening (Fig. 5a), as they would be in a heterogeneous uterine transplantation. The uterus that had been perfused with heparinized saline was transferred to the surgical field from the back-table. The vaginal wall of the excised uterus was anastomosed with the vaginal stump, using the prosthetic valve-suturing technique used in cardiac valve replacement (Fig. 5b). Vascular anastomosis was performed using supermicrosurgery, advanced vascular anastomosis technique, in which $1 \mathrm{~mm}$ or thinner uterine arteries and veins can be anastomosed [24]. The right internal iliac artery of the donor uterus in Case 1 was end-to-end anastomosed with that on the recipient side (diameter: $1.0 \mathrm{~mm}$ ) and the right superficial uterine vein was end-to-end anastomosed with the superficial vesical vein (diameter: $0.9 \mathrm{~mm}$ ) using interrupted sutures (11-0 nylon; Johnson \& Johnson). The superficial vesical vein was used because the right superficial uterine vein was crushed on the pelvic wall side, and the vein on the vesical surface was used as a flap. One artery and one vein were used for perfusion of the uterus in 
Fig. 5 A suction tube was inserted via the vagina; the vaginal stump was identified in the abdominal cavity and opened (a). The vaginal wall and stump were sutured using four or five stitches each in the anterior and posterior regions using 4-0 PDS (b)
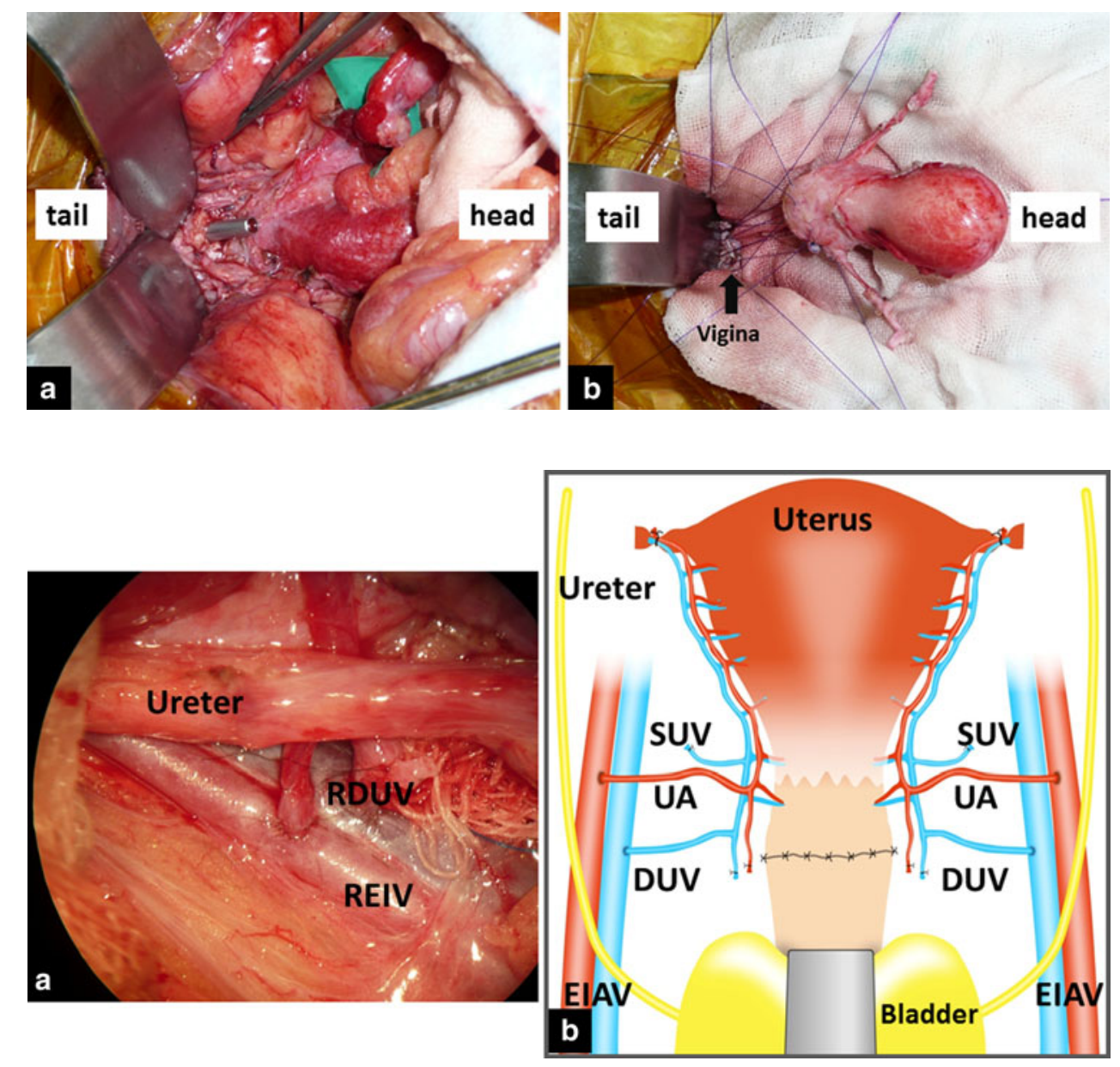

Fig. 6 The right deep uterine vein (RDUV) was end-to-side anastomosed with the right external iliac vein (REIV) (a). Illustration of uterine autotransplantation in a cynomolgus monkey (b). $S U V$ superficial uterine vein, $U A$ uterine artery, $D U V$ deep uterine vein, EIAV external iliac artery and vein
Case 1. The bilateral uterine arteries of the donor uterus in Case 2 were end-to-side anastomosed with the bilateral external iliac arteries (diameter: $0.7 \mathrm{~mm}$ each) using interrupted sutures (11-0 nylon; Johnson \& Johnson). The bilateral deep uterine vein was end-to-side anastomosed with the bilateral external iliac vein (diameter: $0.6 \mathrm{~mm}$ ) using interrupted sutures (11-0 nylon; Johnson \& Johnson) (Fig. 6a). Two arteries and two veins were used for perfusion of the uterus in Case 2 (Fig. 6b). The retroperitoneum and round ligament were attached to fix the uterus to the pelvis. The retroperitoneum was sutured mainly in the vesicouterine and Douglas pouches, but not close to the anastomosed blood vessels. This was followed by round ligament restoration by anastomosis between the round ligament stumps on the pelvic and uterine sides, and the abdomen was closed after ensuring an absence of problems in the abdominal cavity. After the surgery, antibiotics and analgesics were given.

\section{Management after operation}

The animals' general condition and the laparotomy wound were assessed every day. Periodical menstruation was confirmed, and endometrial thickness was inspected by transabdominal ultrasonography. Case 2 underwent a second-look laparotomy at 40 days post-operatively to confirm the engraftment of the uterus. The anesthesia used was identical to that used for primary surgery.

\section{Results}

Surgical parameters

The uterine auto-transplantation of the two cynomolgus monkeys were attempted at different times. Case 1 was conducted with an inspection of anatomical characteristics of the inner genital organs. The total time of anesthetization and surgical operation in Case 1 and 2 was $14 \mathrm{~h}$ $30 \mathrm{~min}$ and $13 \mathrm{~h}$, respectively. The prolonged operation time was required because the organs and blood vessels were far smaller than those in humans. The bleeding volume was small in each case. The time required to excise the uterus was $7 \mathrm{~h} 45 \mathrm{~min}$ and $5 \mathrm{~h} 19 \mathrm{~min}$, respectively. The operation time could be shortened in Case 2 because of the experience gained during Case 1. The back-table 

recovered after vascular anastomosis, but uterine congestion was noted in Case 1 (a) in comparison to the uterus in Case 2 (b)
Fig. 7 The uterine color

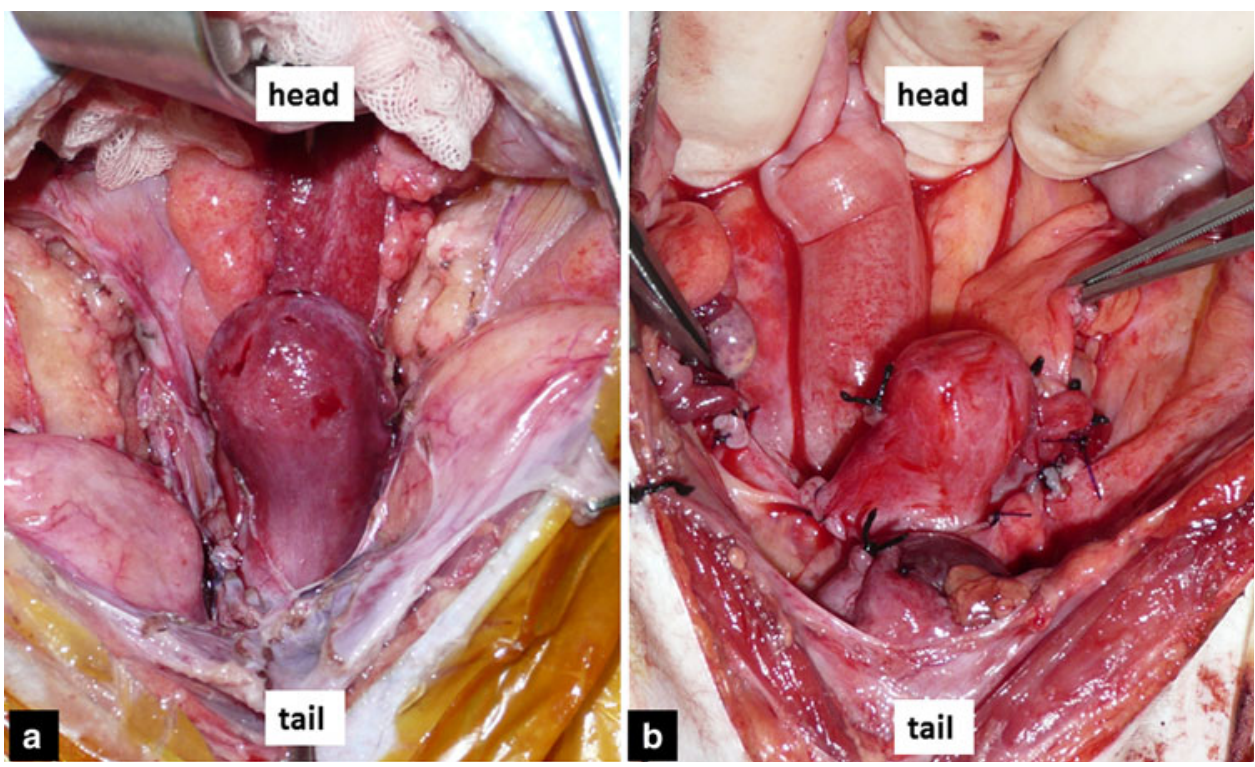

preparation time was 49 and $32 \mathrm{~min}$, respectively. The blood vessel anastomosis time was $3 \mathrm{~h} 55 \mathrm{~min}$, and $4 \mathrm{~h}$ $42 \mathrm{~min}$, respectively. More time was required in Case 2 because the deep uterine vein was identified in the deep region of pelvis and used for anastomosis of the blood vessels, which was technically more difficult. In addition, two arteries and two veins were anastomosed in Case 2, in comparison to one each in Case 1. The total warm ischemic period lasted for $5 \mathrm{~h} 7 \mathrm{~min}$ and $5 \mathrm{~h} 32 \mathrm{~min}$, respectively.

\section{Surgical findings}

Pulsations of uterine arteries and capillary refill of the serosal surface of the uterus were observed after vascular anastomosis and removal of the vascular clamps. The uterine color recovered after vascular anastomosis, but uterine congestion was observed in Case 1 in comparison to the uterus in Case 2 (Fig. 7).

\section{Postoperative progress}

The cynomolgus monkey in Case 1 developed anuria and died due to acute renal failure 2 days after the operation. A small volume of hematoma was observed around the uterus, but it is not believed that death was caused by imperfect anastomosis. The pathological findings of uterus showed normal erythrocytes in the blood vessels, but there was neither fibrin coagulation nor thrombus in the vessel. The ureter was not enlarged, and the possibility that the cynomolgus monkey had post-renal failure was rejected. The reason for its death may be related to acute renal failure due to the effects of the time consuming operation.

Case 2 was in good general condition with appetite, urination, and defecation. Second-look laparoscopy

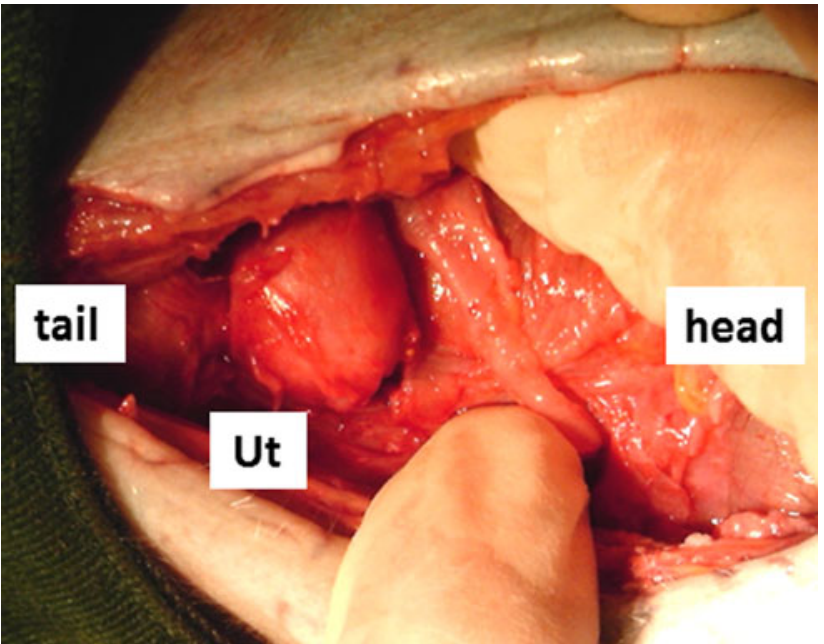

Fig. 8 Second-look laparotomy inspection in Case 2. There was no congestion in the uterus, and it had the characteristic red color of a normal uterus. $U t$ uterus

40 days after the operation confirmed the engraftment of the uterus. The uterus could not be exposed because of extensive adhesion between the rectum, sigmoid colon and small intestine. Therefore, an abdominal section along the previous operation wound was attempted. The intestinal adhesion was separated and the uterus was exposed. No congestion was observed in the uterus, which had the characteristic red color of a normal uterus (Fig. 8). This confirmed the engraftment of the auto-transplanted uterus. The anastomosed blood vessels could not be inspected because of extensive intestinal adhesion. The endometrial thickness was inspected periodically by transabdominal ultrasonography, and endometrial proliferation was observed. Periodical menstruation was confirmed on 
139 days after operation, and thereafter, the monkey remained in good general condition.

\section{Discussion}

We performed experimental uterine auto-transplantation in two cynomolgus monkeys. Hysterectomy was similar to the procedure used in humans because the anatomy in the pelvis was almost the same as that in humans. However, prolonged operation time was required because the organs and blood vessels were far smaller than those in humans.

A vascularized uterus with the vaginal wall is required for uterine transplantation because pregnancy cannot be achieved after non-vascularized uterine auto-transplantation in rhesus monkeys [25]. Therefore, the selection of vascular grafts is important for uterine perfusion. Previous studies have assessed the uterine blood supply and perfusion with uterine and ovarian vessels [26-29].

The main feeder arteries of the uterus are the uterine and ovarian arteries, which branch from the internal iliac artery and the aorta, respectively. Blood flow was supplied only by the uterine artery in this experiment. The uterine artery is present on bilateral sides, but a unilateral artery may be sufficient to supply blood flow to the uterus, although this is uncertain for the uterus during pregnancy. Preparation of a vascular flap with the inferior epigastric artery may be required if the vascular pedicle length is insufficient. The uterine artery is not dissected or excised in a simple total hysterectomy, but this procedure with a uterine arterial pedicle is relatively straightforward because the uterine artery is located in a fairly shallow site in the pelvic cavity.

The uterine venous system is mainly composed of the superficial and deep uterine veins and the ovarian veins. Maintenance of the venous circulation is very important to avoid uterine congestion, thrombus formation, and subsequent uterine necrosis. There was a marked difference in the reconstruction of the venous system between the two procedures because the deep uterine vein was used as a vascular pedicle in Case 2. The human deep uterine vein is located in the deep pelvic region, deeper than the superficial uterine vein. It is thick and participates in the constitution of the cardinal ligament. This vein was also present deep in the pelvic cavity of the cynomolgus monkey. The transplanted uterus in Case 2 had a favorable uterine color after vascular anastomosis in comparison to that in Case 1, suggesting the importance of the deep uterine vein for venous circulation in the uterus to prevent congestion. Therefore, a donor uterus vascularized by the deep uterine vein may be desirable to prevent congestion if uterus transplantation is performed in humans. The excision of this vein as a vascular pedicle increases the difficulty of the surgery and makes the procedure more invasive because the vein is located in the deep region of pelvis and can only be reached via processing of the surrounding tissues. Preparation of a vascular flap with the inferior epigastric vein may be used when the vascular pedicle length is insufficient, with care taken to avoid congestion due to vascular twisting.

The need for inclusion of the vagina in uterine transplantation is questionable. Vaginal reconstruction may be necessary because it is the excretory pathway of uterine secretions, including menstruation, and the vagina supports the uterus in the pelvis. Furthermore, the vagina allows direct external access to the intrauterine region, which enables endometrial biopsy, transvaginal ultrasonography, embryo transfer, and sexual activity, increasing the possibility of natural pregnancy.

The uterine transplantation procedure in recipients consists of anastomosis of the vaginal canal with the vascularized donor uterus and vaginal wall, vascular anastomosis, and restoration of the round ligament and retroperitoneum. The main uterus-supporting ligaments are the round ligament, vesicocervical ligament, cardinal ligament, and uterosacral ligament in humans. Uterine transplantation in a recipient may require fixation of the grafted uterus in the pelvic cavity because the mobility of the uterus in the pelvic cavity may cause twisting of the anastomosed blood vessels. In a radical trachelectomy, the uterine cervix is excised after cutting all the ligaments, and the uterine body is anastomosed to the upper vaginal cuff $[30,32]$. Pregnancy and delivery are possible when only the round ligament is restored, but ligament restoration, anastomosis with the vaginal wall and suture of the broad ligament are important for fixation of the grafted uterus to the pelvis.

Hysterectomy that differs from the low-invasive simple total hysterectomy is necessary to prepare the deep uterine vein for grafting. The procedure comes close to a radical hysterectomy because the processing of surrounding tissues to the depth of the deep uterine vein is required and the resection length of the vaginal wall is elongated. Therefore, a 14-mm vaginal wall was excised in Case 2. Blood loss also increases and the operation time is prolonged in a radical hysterectomy. Moreover, dysuria may occur due to ureteral and urinary bladder injury or pelvic nerve plexus and hypogastric nerve injury. All of these factors increase the invasiveness of the procedure. Therefore, living donor uterine transplantation may carry a risk of complications that reduce the postoperative quality-of-life of the donor. Sufficient explanation of this risk will be necessary for obtaining informed consent.

Vascularized uterine auto-transplantation in primates other than humans has previously been reported in baboons, which are used as a model because the anatomy of the uterus is similar to that of humans: the uterine cervix 
is linear and endometrial sampling, embryo transfer, and hysteroscopy can easily be performed [23, 33]. The uterine cervix is bent in cynomolgus monkeys, and making an approach via the cervical canal is difficult. Consequently, embryo transfer is performed via a fallopian tube or muscle layer through the uterine wall in many cases [31, 34]. Embryo transfer in the current model would have to be performed via the muscle layer through the uterine wall because the bilateral fallopian tubes were resected. Therefore, a procedure for conservation of the fallopian tubes in the transplanting uterus should be investigated to maintain the choice of laparoscopic trans-fallopian tube embryo transfer. IVF and embryo transfer would be performed in this uterine auto-transplantation model in an attempt to achieve pregnancy and delivery.

Even if uterine auto-transplantation model in cynomolgus monkey is established technically, there are clearly many problems other than surgical techniques to overcome in heterogeneous uterine transplantation or on the assumption of clinical application in humans. These include ischemia-reperfusion injury, immunosuppression, rejection reactions, evaluation of the grafted uterus, and management of pregnancy and delivery. Further investigation of these problems and accumulation of relevant basic data will be required for establishment of a uterine transplantation model in primates.

Experimental reproductive organ transplantation and cryopreservation have been successful in animal models as well as in humans for fertility treatment. Regarding the uterus cryopreservation, there has been a recent report that uterus cryopreservation improves the availability of uterus for transplantations purposes in the future [35]. Long-term preservation of uterus can, therefore, be important not only for restoring fertility, but also for transplantation surgery.

In conclusion, uterine auto-transplantation experiments were performed in cynomolgus monkeys. To establish an experimental model of uterine transplantation in nonhuman primates may be possible using the developing new surgical techniques described in this article, but many other problems remain to be overcome. Therefore, accumulation of basic experimental data in uterine transplantation studies using primates is required prior to the performance of the procedure in humans.

Acknowledgments This study was supported by the Japan Society for the Promotion of Science (Grant-in-Aid for Young Scientists A); Japan Interaction in Science and Technology Forum Foundation; New Energy and Industrial Technology Development Organization; and Japanese funding program for next generation world leading researchers.

Conflict of interest The authors declare that they have no conflict of interest.
Open Access This article is distributed under the terms of the Creative Commons Attribution Noncommercial License which permits any noncommercial use, distribution, and reproduction in any medium, provided the original author(s) and source are credited.

\section{References}

1. Campagne DM (2006) Should fertilization treatment start with reducing stress? Hum Reprod 21:1651-1658

2. Nakashu H, Herdiman J (2007) Surrogacy. J Obstet Gynaecol 27:246-251

3. Sonoda Y, Abu-Rustum NR, Gemignani ML et al (2004) A fertility-sparing alternative to radical hysterectomy: how many patients may be eligible? Gynecol Oncol 95:534-538

4. Quinn MA, Benedet JL, Odicino F et al (2006) Carcinoma of the cervix uteri. FIGO $26^{\text {th }}$ annual report on the results on treatment in gynaecological cancer. Int J Gynaecol Obstet 95:S43-S103

5. Creasman WT, Odicino F, Barrett T et al (2006) Carcinoma of the corpus uteri. FIGO 26th annual report on the results on treatment in gynaecological cancer. Int $\mathrm{J}$ Gynaecol Obstet 95:S105-S143

6. Marshall LM, Spiegelman D, Barbieri RL et al (1997) Variation in the incidence of uterine leiomyoma among premenopausal women by age and race. Obstet Gynecol 90:967-973

7. Saravelous SH, Cocksedge KA, Li TC (2008) Prevalence and diagnosis of congenital uterine anomalies in women with rproductive failure: a critical appraisal. Hum Reprod Update 14:415-429

8. Bryan AL, Nigro JA, Counseller VS (1949) One hundred cases of congenital absence of the vagina. Surg Gynecol Obstet 88:79-86

9. Fageeh W, Raffa H, Jabbad H et al (2002) Transplantation of the human uterus. Int J Gynecol Obstet 7:245-251

10. McKay DB, Josephson MA (2006) Pregnancy in recipients of solid organs-effects on mother and child. $N$ Eng $J$ Med 354:1281-1293

11. Devauchelle B, Badet L, Lengele B et al (2006) First human face allograft: early report. Lancet 368:203-209

12. Silló-Seidl G (1975) The first transplantation of a Fallopian tube of frozen material in woman. Int J Fertil 20(2):106-108

13. Cohen BM (1980) Fallopian tube transplantation and its future. Clin Obstet Gynecol 23(4):1275-1292

14. Racho El-Akouri R, Kurlberg G, Brännström M (2003) Successful uterine transplantation in the mouse: pregnancy and postnatal development of offspring. Hum Reprod 18:2018-2023

15. Jiga LP, Lupu CM, Zoica BS et al (2003) Experimental model of heterotopic uterus transplantation in the laboratory rat. Microsurgery 23:246-250

16. Wranning CA, Akhi SN, Kurlberg G et al (2008) Uterus transplantation in the rat: model development, surgical learning and morphological evaluation of healing. Acta Obstet Gynecol Scand 87:1239-1247

17. Sieunarine K, Zakaria FBP, Boyle DCM et al (2005) Possibilities for fertility restoration: a new surgical technique. Int Surg 90:249-256

18. Avison DL, DeFaria W, Tryphonopoulos P et al (2009) Heterotopic uterus transplantation in a swine model. Transplantation 88:465-469

19. Ramirez ER, Ramirez DK, Pillari VT et al (2008) Modified uterine transplant procedure in the sheep model. J Minim Invasive Gynecol 15:311-314

20. Enskog A, Johannesson L, Chai DC et al (2010) Uterus transplantation in the baboon: methodology and long-term function after auto-transplantation. Hum Reprod 25:1980-1987 
21. Milliez J (2009) Uterine transplantation. Int J Gynecol Obstet 106:270

22. Del Priore G, Schlatt S, Malanowska-Stega J (2008) Uterus transplant techniques in primates: 10 years' experience. Exp Clin Transplant 6(1):87-94

23. Nyachieo A, Chai DC, Deprest J et al (2007) The baboon as a research model for the study of endometrial biology, uterine receptivity and embryo implantation. Gynecol Obstet Invest 64:149-155

24. Koshima I, Yamamoto T, Narushima M et al (2010) Perforator flaps and supermicrosurgery. Clin Plast Surg 37(4):683689

25. Scott JR, Pitkin RM, Yannone ME (1971) Transplantation of the primate uterus. Surg Gynecol Obstet 133:414-418

26. Sieunarine K, Boyle DC, Corless DJ et al (2006) Pelvic vascular prospects for uterine transplantation. Int Surg 91:217-222

27. Moxey P, Sieunarine K, Cox J et al (2006) Pulse oximetry and perfusion index measurement to assess uterine perfusion and viability. Int Surg 91:223-227

28. Sieunarine K, Moxey P, Boyle DCM et al (2005) Selective vessel ligation in the pelvis: an invaluable tool in certain surgical procedures. Int J Gynecol Cancer 15:968-973
29. Cicinelli E, Einer-Jensen N, Barba B et al (2004) Blood to the corneal area of the uterus is mainly supplied from the ovarian artery in the follicular phase and from the uterine artery in the luteal phase. Hum Reprod 19:1003-1008

30. Ramirez PT, Schmeler KM, Soliman PT, Frumovitz M (2008) Fertility preservation in patients with early cervical cancer: radical trachelectomy. Gynecol Oncol 110:S25-S28

31. Summers PM, Shephard AM, Taylo CT et al (1987) The effects of cryopreservation and transfer on embryonic development in the common marmoset monkey, Callithrix jacchus. J Reprod Fert 79:241-250

32. Wranning C, Mölne J, Racho El-Akouri R et al (2005) Short-term ischaemic storage of human uterine myometrium-basic studies towards uterine transplantation. Hum Reprod 20:2744-2763

33. Del Priore G, Stega J, Sieunarine K et al (2007) Human uterus retrieval from a multi-organ donor. Obstet Gynecol 109:101-104

34. Brännström M, Wranning C, Altchek A (2010) Experimental uterus transplantation. Hum Reprod Update 16:329-345

35. Dittrich R, Beckmann MW, Mueller A, Binder H, Hoffmann I, Maltaris T (2010) Uterus cryopreservation: maintenance of uterine contractility by the use of different cryoprotocols. Reprod Domest Anim 45(1):86-91 